\title{
A Projection-Type Method for Multivalued Variational Inequality
}

\author{
Changjie Fang, Shenglan Chen, and Jiming Zheng \\ Institute of Applied Mathematics, Chongqing University of Posts and Telecommunications, Chongqing 400065, China \\ Correspondence should be addressed to Changjie Fang; fangcj@cqupt.edu.cn
}

Received 18 April 2013; Revised 5 August 2013; Accepted 19 August 2013

Academic Editor: Shawn X. Wang

Copyright (C) 2013 Changjie Fang et al. This is an open access article distributed under the Creative Commons Attribution License, which permits unrestricted use, distribution, and reproduction in any medium, provided the original work is properly cited.

\begin{abstract}
We propose a projection-type method for multivalued variational inequality. The iteration sequence generated by the algorithm is proven to be globally convergent to a solution, provided that the multivalued mapping is continuous with nonempty compact convex values. Moreover, we present a necessary and sufficient condition on the nonemptiness of the solution set. Preliminary computational experience is also reported.
\end{abstract}

\section{Introduction}

We consider the following multivalued variational inequality, denoted by $\operatorname{GVI}(T, K)$ to find $x^{*} \in K$ and $w^{*} \in T\left(x^{*}\right)$ such that

$$
\left\langle w^{*}, y-x^{*}\right\rangle \geq 0, \quad \forall y \in K,
$$

where $K$ is a nonempty closed convex set in $\mathbb{R}^{n}, T$ is a multivalued mapping from $K$ into $\mathbb{R}^{n}$ with nonempty values, and $\langle\cdot, \cdot\rangle$ and $\|\cdot\|$ denote the inner product and norm in $\mathbb{R}^{n}$, respectively.

Projection-type algorithms have been extensively studied in the literature; see [1-8] and the references therein. Reference [2] proposes a subgradient extragradient algorithm for solving single-valued variational inequality in which the next iterate is a projection onto a halfspace whose bounding hyperplane supports the feasible set $K$ at a certain point. Reference [6] proposes a projection method for variational inequality problems in which the hyperplane strictly separates the current iterate from the solution set of (1). Theory and algorithm of multivalued variational inequality have been much studied in the literature [1,9-18]. Various algorithms for computing the solution of (1) are proposed. The well-known proximal point algorithm [19] requires the multivalued mapping $T$ to be monotone. Relaxing the monotonicity assumption, [12] proposed the double projection algorithm for solving (1); also see [5]. Assume that $T$ is pseudomonotone; [20] described a combined relaxation method for solving (1); see also [21]. Recently, [22] proposed an extragradient method for generalized variational inequality. In [22], the next iterate is the projection of the current iterate onto the feasible set $K$; also see [23].

In this paper, we introduce a projection-type method for multivalued variational inequality in which the next iterate is a projection of the initial iterate onto intersection of two halfspaces containing the solution set. We obtain a global convergence theorem, assuming that $T$ is pseudomonotone on $K$ with respect to the solution set; see (3) in the following. Moreover, we show that the iterative sequence diverges if and only if the solution set is empty. We also present numerical results of the proposed method. Now let us compare our algorithm with algorithms in $[5,12,22,23]$. First, the next iterate in our method relates to the initial point. In $[5,12]$, the next iterate is the projection of the current iterate onto the intersection of the hyperplane and the feasible set $K$. Secondly, the next iterate in our method is a projection of the initial point onto the intersection of the two hyperplanes and the feasible set $K$. In addition, our Armijo-type linesearch procedure is also different from those in [12, 22, 23].

The organization of this paper is as follows. In the next section, we present the algorithm details and some lemmas. We prove several preliminary results for convergence analysis in Section 3. Numerical results are reported in the last section. 


\section{Algorithms}

Let us recall the definition of continuous multivalued mapping. $T$ is said to be upper semicontinuous at $x \in K$ if, for every open set $V$ containing $T(x)$, there is an open set $U$ containing $x$ such that $T(y) \subset V$ for all $y \in K \cap U . T$ is said to be lower semicontinuous at $x \in K$ if, given any sequence $\left\{x_{k}\right\}$ converging to $x$ and any $y \in T(x)$, there exists a sequence $y_{k} \in T\left(x_{k}\right)$ that converges to $y$. T is said to be continuous at $x \in T$ if, it is both upper semicontinuous and lower semicontinuous at $x$. If $T$ is single valued, then both upper semicontinuity and lower semicontinuity reduce to the continuity of $T$.

$T$ is called pseudomonotone on $K$ in the sense of Karamardian [24], if, for any $x, y \in K$,

$$
\begin{aligned}
\langle v, x-y\rangle & \geq 0, \quad \forall v \in T(y) \Longrightarrow\langle u, x-y\rangle \\
& \geq 0, \quad \forall u \in T(x) .
\end{aligned}
$$

Let $S$ be the solution set of (1), that is, those points $x^{*} \in K$ satisfying (1). Throughout this paper, we assume that the solution set $S$ of the problem (1) is nonempty and $T$ is continuous on $K$ with nonempty compact convex values satisfying the following property:

$$
\langle\zeta, y-x\rangle \geq 0, \quad \forall y \in K, \zeta \in T(y), \forall x \in S .
$$

The property (3) holds if $T$ is pseudomonotone on $K$.

Let $\Pi_{K}$ denote the projector onto $K$, and let $\mu>0$ be a parameter.

Proposition 1. $x \in K$ and $\xi \in T(x)$ solves the problem (1) if and only if

$$
r_{\mu}(x, \xi):=x-\Pi_{K}(x-\mu \xi)=0 .
$$

Algorithm 2. Choose $x_{0} \in K$ and three parameters $\sigma>$ $0, \mu \in(0,1 / \sigma)$, and $\gamma \in(0,1)$. Set $i=0$.

Step 1. If $r_{\mu}\left(x_{i}, w\right)=0$ for some $w \in T\left(x_{i}\right)$, stop; else take arbitrarily $w_{i} \in T\left(x_{i}\right)$.

Step 2. For every positive integer $k$, let $y_{k}=$ $\Pi_{T\left(x_{i}-\gamma^{k} r_{\mu}\left(x_{i}, w_{i}\right)\right)}\left(w_{i}\right)$.

Step 3. Let $k_{i}$ be the smallest nonnegative integer $k$ satisfying

$$
\left\langle y_{k}, r_{\mu}\left(x_{i}, w_{i}\right)\right\rangle \geq \sigma\left\|r_{\mu}\left(x_{i}, w_{i}\right)\right\|^{2} .
$$

Set $\eta_{i}=\gamma^{k_{i}}$ and $z_{i}=x_{i}-\eta_{i} r_{\mu}\left(x_{i}, w_{i}\right)$.

Step 4. Compute $x_{i+1}:=\prod_{H_{i}^{1} \cap H_{i}^{2} \cap K}\left(x_{0}\right)$, where

$$
\begin{aligned}
& H_{i}^{1}:=\left\{x \in R^{n}:\left\langle y_{k_{i}}, x-z_{i}\right\rangle \leq 0\right\}, \\
& H_{i}^{2}:=\left\{x \in R^{n}:\left\langle x-x_{i}, x_{0}-x_{i}\right\rangle \leq 0\right\} .
\end{aligned}
$$

Let $i:=i+1$, and go to Step 1 .
Remark 3. Let us compare the previous algorithm with Algorithm 3.1 in [11]. First, the parameter $\sigma>0$ is required to be strictly less than 1 , and $\mu$ is assumed to be equal to 1 in their Algorithm 3.1. In our Algorithm 2, the parameter $\sigma$ can take any positive scalar and $\mu \in(0,1 / \sigma)$. Secondly, since $T$ has compact convex values, $T$ has closed convex values. Therefore, $y_{k}$ in Step 2 of our algorithm is uniquely determined by $k$. Hence, it is easy to compute the value of $y_{k}$ satisfying (5). In Step 1 of their Algorithm 3.1, since F is a multivalued mapping, it is very difficult in practice to compute the value of $\zeta^{k}$ satisfying $\zeta^{k} \in F\left(x^{k}-\gamma^{m} r\left(x^{k}, \xi^{k}\right)\right)$ and $\left\langle\zeta^{k}, r\left(x^{k}, \xi^{k}\right)\right\rangle \geq \sigma\left\|r\left(x^{k}, \xi^{k}\right)\right\|^{2}$ at the same time. In addition, we report numerical results concerning our algorithm, while [11] does not present numerical experiments for the proposed algorithm. Finally, we compare the performance of our algorithm and Algorithm 3.1 in [11] (see Table 4).

Lemma 4. The sequence $\left\{y_{k}\right\}$ generated in Step 2 has the following properties:

$$
y_{k} \in T\left(x_{i}-\gamma^{k} r_{\mu}\left(x_{i}, w_{i}\right)\right), \quad \lim _{k \rightarrow \infty} y_{k}=w_{i} .
$$

Proof. See Lemma 2.1 in [5].

Lemma 5. For every $x \in K$ and $w \in T(x)$,

$$
\left\langle w, r_{\mu}(x, w)\right\rangle \geq \mu^{-1}\left\|r_{\mu}(x, w)\right\|^{2} .
$$

Proof. See Lemma 2.3 in [5].

We show that Algorithm 2 is well defined and implementable.

Lemma 6. If $r_{\mu}\left(x_{i}, w_{i}\right) \neq 0$, there exists $k$ satisfying (5).

Proof. In view of Lemma 4, we have $\lim _{k \rightarrow \infty} y_{k}=w_{i}$. Therefore,

$$
\begin{aligned}
\lim _{k \rightarrow \infty}\left\langle y_{k}, r_{\mu}\left(x_{i}, w_{i}\right)\right\rangle & =\left\langle w_{i}, r_{\mu}\left(x_{i}, w_{i}\right)\right\rangle \\
& \geq \mu^{-1}\left\|r_{\mu}\left(x_{i}, w_{i}\right)\right\|^{2} \\
& >\sigma\left\|r_{\mu}\left(x_{i}, w_{i}\right)\right\|^{2},
\end{aligned}
$$

where the first inequality follows from Lemma 5 and the second one follows from $\mu^{-1}>\sigma$ and $r_{\mu}\left(x_{i}, w_{i}\right) \neq 0$.

Lemma 7. Let $K$ be a closed convex subset of $\mathbb{R}^{n}$. For any $x, y \in$ $\mathbb{R}^{n}$ and $z \in K$, the following statements hold:

(i) $\left\langle\Pi_{K}(x)-x, z-\Pi_{K}(x)\right\rangle \geq 0$,

(ii) $\left\|\Pi_{K}(x)-\Pi_{K}(y)\right\|^{2} \leq\|x-y\|^{2}-\| \Pi_{K}(x)-x+y-$ $\Pi_{K}(y) \|^{2}$.

Proof. See [25].

Lemma 8. Let $x^{*} \in S$ and $h_{i}(x):=\left\langle y_{k_{i}}, x-z_{i}\right\rangle$. If $r_{\mu}\left(x_{i}, w_{i}\right) \neq 0$, then the hyperplane

$$
\partial H_{i}^{1}=\left\{x \in \mathbb{R}^{n}: h_{i}(x)=0\right\}
$$

strictly separates $x_{i}$ and the solution set $S$. 
Proof. Since $z_{i}=x_{i}-\eta_{i} r_{\mu}\left(x_{i}, w_{i}\right)$

$$
\begin{aligned}
h_{i}\left(x_{i}\right) & =\left\langle y_{k_{i}}, x_{i}-z_{i}\right\rangle \\
& =\eta_{i}\left\langle y_{k_{i}}, r_{\mu}\left(x_{i}, w_{i}\right)\right\rangle \\
& \geq \sigma \eta_{i}\left\|r_{\mu}\left(x_{i}, w_{i}\right)\right\|^{2} \\
& >0,
\end{aligned}
$$

where the first inequality follows from (5) and the last one follows from $\left.r_{\mu}\left(x_{i}, w_{i}\right)\right) \neq 0$. Since $T$ satisfies the property (3), $h_{i}\left(x^{*}\right) \leq 0$.

Lemma 9. If $S \neq \emptyset$, then $S \subset H_{i}^{1} \cap H_{i}^{2} \cap K$.

Proof. It follows from Lemma 8 that $S \subset H_{i}^{1} \cap K$. Next, it is sufficient to prove that $S \subset H_{i}^{2}$ for all $i \geq 0$. The proof will be given by induction.

Obviously, $S \subset H_{0}^{2}=\mathbb{R}^{n}$. Suppose that

$$
S \subset H_{i}^{2}, \quad \text { for } i=m \geq 0 \text {. }
$$

Then, $S \subset H_{m}^{1} \cap H_{m}^{2} \cap K$.

Let $x^{*} \in S$. Since

$$
x_{m+1}=\Pi_{H_{m}^{1} \cap H_{m}^{2} \cap K}\left(x_{0}\right),
$$

it follows from Lemma 7 (i) that

$$
\left\langle x^{*}-x_{m+1}, x_{0}-x_{m+1}\right\rangle \leq 0 .
$$

Thus, $S \subset H_{m+1}^{2}$. Therefore, we obtain that $S \subset H_{i}^{2}$ for all $i$.

Lemma 10. Let $K \subset \mathbb{R}^{n}$ be a nonempty bounded closed convex set, and let the mapping $T: K \rightarrow 2^{\mathbb{R}^{n}}$ be lower semicontinuous with nonempty closed convex values; then, the solution set $S$ of $G V I(T, K)$ is nonempty.

Proof. See Lemma 2.9 in [22].

The following lemma says that if the solution set $S$ is empty, then $H_{i}^{1} \cap H_{i}^{2} \cap K$ is a nonempty set, which implies the feasibility of Algorithm 2.

Lemma 11. Let $T: K \rightarrow 2^{\mathbb{R}^{n}}$ be continuous with nonempty compact convex values on $K$, and suppose that $S=\emptyset$; then, $H_{i}^{1} \cap H_{i}^{2} \cap K \neq \emptyset$ for all $i$.

Proof. On the contrary, suppose that there exists $i_{0} \geq 0$ such that $H_{i_{0}}^{1} \cap H_{i_{0}}^{2} \cap K=\emptyset$. Then, there exists a positive number $M$ such that

$$
\left\{x_{i}: 0 \leq i \leq i_{0}\right\} \subseteq B\left(x_{0}, M\right)
$$

where

$$
B\left(x_{0}, M\right):=\left\{x \in R^{n}:\left\|x-x_{0}\right\| \leq M\right\} .
$$

Since $T(x)$ is continuous with compact values, Proposition 3.11 in [26] implies that $\left\{T\left(x_{i}\right): 0 \leq i \leq i_{0}\right\}$ is a bounded set, and so $\left\{x_{i}-\mu w_{i}: w_{i} \in T\left(x_{i}\right), 0 \leq i \leq i_{0}\right\}$ is bounded. Without loss of generality, we assume that

$$
\left\{x_{i}-\mu w_{i}: w_{i} \in T\left(x_{i}\right), 0 \leq i \leq i_{0}\right\} \subseteq B\left(x_{0}, M\right) .
$$

Consider the variational inequality $\operatorname{GVI}(T, C)$, where

$$
C=K \cap B\left(x_{0}, 2 M\right) \text {. }
$$

It follows from Lemma 10 that the solution set of $\operatorname{GVI}(T, C)$, denoted by $S_{1}$, is nonempty. We denote the three sequences $\left\{H_{i}^{1}\right\},\left\{H_{i}^{2}\right\}$, and $\left\{x_{i}\right\}$ by $\left\{\widetilde{H}_{i}^{1}\right\},\left\{\widetilde{H}_{i}^{2}\right\}$, and $\left\{\tilde{x}_{i}\right\}$, respectively, when Algorithm 2 is applied to $\operatorname{GVI}(T, C)$ with starting point $x_{0}$. We claim that

(i) the set $\left\{\tilde{x}_{i}\right\}$ has at least $i_{0}+1$ elements;

(ii) $x_{i}=\widetilde{x}_{i}, H_{i}^{1}=\widetilde{H}_{i}^{1}$, and $H_{i}^{2}=\widetilde{H}_{i}^{2}$ for $0 \leq i \leq i_{0}$;

(iii) $x_{i_{0}}$ is not a solution of $\operatorname{GVI}(T, C)$.

Items (i) and (iii) are obvious. Next we prove the item (ii). It is sufficient to prove that

$$
\Pi_{K}\left(x_{i}-\mu w_{i}\right)=\Pi_{C}\left(x_{i}-\mu w_{i}\right),
$$

where $w_{i} \in T\left(x_{i}\right), 0 \leq i \leq i_{0}$.

Since

$$
\begin{aligned}
\left\|x_{0}-\Pi_{K}\left(x_{i}-\mu w_{i}\right)\right\| \leq & \left\|x_{0}-\Pi_{K}\left(x_{0}\right)\right\| \\
& +\left\|\Pi_{K}\left(x_{0}\right)-\Pi_{K}\left(x_{i}-\mu w_{i}\right)\right\| \\
& \leq\left\|x_{0}-x_{i_{0}}\right\|+\left\|x_{0}-\left(x_{i}-\mu w_{i}\right)\right\| \\
& \leq 2 M,
\end{aligned}
$$

where the second inequality follows from $x_{i_{0}} \in K$ and Lemma 7 (ii), so $\Pi_{K}\left(x_{i}-\mu w_{i}\right) \in B\left(x_{0}, 2 M\right)$, and hence, $\Pi_{K}\left(x_{i}-\mu w_{i}\right) \in K \cap B\left(x_{0}, 2 M\right)=C$. Therefore,

$$
\Pi_{K}\left(x_{i}-\mu w_{i}\right)=\Pi_{C}\left(x_{i}-\mu w_{i}\right) .
$$

Since $S_{1} \neq \emptyset$, it follows from Lemma 9 that $\widetilde{H}_{i}^{1} \cap \widetilde{H}_{i}^{2} \cap C \neq \emptyset$. Therefore, $H_{i}^{1} \cap H_{i}^{2} \cap K \neq \emptyset$ for $0 \leq i \leq i_{0}$, which contradicts the supposition that $H_{i_{0}}^{1} \cap H_{i_{0}}^{2} \cap K=\emptyset$.

\section{Main Results}

Theorem 12. Let $T: K \rightarrow 2^{\mathbb{R}^{n}}$ be continuous with nonempty compact convex values on $K$ satisfying condition (3). Suppose that Algorithm 2 generates an infinite sequence $\left\{x_{i}\right\}$. If the solution set $S$ of $\operatorname{GVI}(T, K)$ is nonempty, then $\left\{x_{i}\right\}$ globally converges to a solution $x^{*}$ of $\operatorname{GVI}(T, K)$ satisfying $x^{*}=$ $\Pi_{S}\left(x_{0}\right)$.

Proof. Since $x_{i+1}=\Pi_{H_{i}^{1} \cap H_{i}^{2} \cap K}\left(x_{0}\right)$, by Lemma 9 and the definition of projection, it follows that

$$
\left\|x_{i+1}-x_{0}\right\| \leq\left\|x^{*}-x_{0}\right\|, \quad \forall x^{*} \in S .
$$

Therefore, $\left\{x_{i}\right\}$ is a bounded sequence. 
Since $x_{i+1} \in H_{i}^{1} \cap H_{i}^{2} \cap K \subset H_{i}^{2}, P_{H_{i}^{2}}\left(x_{i+1}\right)=x_{i+1}$. Since

$$
\left\langle z-x_{i}, x_{0}-x_{i}\right\rangle \leq 0, \quad \forall z \in H_{i}^{2},
$$

it follows that

$$
x_{i}=\Pi_{H_{i}^{2}}\left(x_{0}\right) .
$$

Thus, it follows from Lemma 7 (ii) that

$$
\begin{aligned}
\left\|\Pi_{H_{i}^{2}}\left(x_{i+1}\right)-\Pi_{H_{i}^{2}}\left(x_{0}\right)\right\|^{2} \\
\quad \leq\left\|x_{i+1}-x_{0}\right\|^{2} \\
\quad-\left\|\Pi_{H_{i}^{2}}\left(x_{i+1}\right)-x_{i+1}+x_{0}-\Pi_{H_{i}^{2}}\left(x_{0}\right)\right\|^{2} ;
\end{aligned}
$$

that is,

$$
\left\|x_{i+1}-x_{i}\right\|^{2} \leq\left\|x_{i+1}-x_{0}\right\|^{2}-\left\|x_{i}-x_{0}\right\|^{2} .
$$

Thus, the sequence $\left\{\left\|x_{i}-x_{0}\right\|\right\}$ is nondecreasing and bounded and hence convergent, which implies that

$$
\lim _{i \rightarrow \infty}\left\|x_{i+1}-x_{i}\right\|^{2}=0 .
$$

On the other hand, since

$$
\Pi_{H_{i}^{1}}\left(x_{i}\right)=x_{i}-\left[\frac{\left\langle y_{k_{i}}, x_{i}-z_{i}\right\rangle}{\left\|y_{k_{i}}\right\|^{2}}\right] y_{k_{i}}
$$

and since $x_{i+1} \in H_{i}^{1}$, we have

$$
\begin{aligned}
\left\|x_{i}-x_{i+1}\right\| \geq\left\|x_{i}-\Pi_{H_{i}^{1}}\left(x_{i}\right)\right\| \\
=\frac{\left\langle y_{k_{i}}, x_{i}-z_{i}\right\rangle}{\left\|y_{k_{i}}\right\|} \\
\geq \sigma \eta_{i} \frac{\left\|r_{\mu}\left(x_{i}, w_{i}\right)\right\|^{2}}{\left\|y_{k_{i}}\right\|},
\end{aligned}
$$

where the second inequality follows from (5). Therefore, it follows from (27) that

$$
\lim _{i \rightarrow \infty} \eta_{i} \frac{\left\|r_{\mu}\left(x_{i}, w_{i}\right)\right\|^{2}}{\left\|y_{k_{i}}\right\|}=0 .
$$

Since $T$ is continuous with compact values, Proposition 3.11 in [26] implies that $\left\{T\left(x_{i}\right)\right\}$ is a bounded set, and so the sequences $\left\{w_{i}\right\}$ and $\left\{z_{i}\right\}$ are bounded. Thus, the continuity of $T$ implies that $\left\{T\left(z_{i}\right)\right\}$ is a bounded set. Therefore, $\left\{y_{k_{i}}\right\}$ is bounded. It follows that

$$
\lim _{i \rightarrow \infty} \eta_{i}\left\|r_{\mu}\left(x_{i}, w_{i}\right)\right\|^{2}=0 .
$$

By the boundedness of $\left\{x_{i}\right\}$, there exists a convergent subsequence $\left\{x_{i_{j}}\right\}$ converging to $\bar{x}$.
If $\bar{x}$ is a solution of the problem (1), we show next that the whole sequence $\left\{x_{i}\right\}$ converges to $\bar{x}$. Let $x^{*}=\Pi_{S}\left(x_{0}\right)$. Since $x^{*} \in S$, by Lemma 9 ,

$$
x^{*} \in H_{i_{j}-1}^{1} \cap H_{i_{j}-1}^{2} \cap K, \quad \forall j \geq 1 .
$$

Therefore,

$$
\left\|x_{i_{j}}-x_{0}\right\| \leq\left\|x^{*}-x_{0}\right\|
$$

Thus,

$$
\begin{aligned}
\left\|x_{i_{j}}-x^{*}\right\|^{2}= & \left\|x_{i_{j}}-x_{0}\right\|^{2}+\left\|x_{0}-x^{*}\right\|^{2} \\
& +2\left\langle x_{i_{j}}-x_{0}, x_{0}-x^{*}\right\rangle \\
\leq & 2\left\|x_{0}-x^{*}\right\|^{2}+2\left\langle x_{i_{j}}-x_{0}, x_{0}-x^{*}\right\rangle .
\end{aligned}
$$

Letting $j \rightarrow \infty$ in (34), we have

$$
\begin{aligned}
\left\|\bar{x}-x^{*}\right\|^{2} & \leq 2\left\|x_{0}-x^{*}\right\|^{2}+2\left\langle\bar{x}-x_{0}, x_{0}-x^{*}\right\rangle \\
& =2\left\langle\bar{x}-x^{*}, x_{0}-x^{*}\right\rangle \\
& \leq 0
\end{aligned}
$$

where the last inequality follows from Lemma 7 (i) and the fact that $x^{*}=\Pi_{S}\left(x_{0}\right)$ and $\bar{x} \in S$. Therefore,

$$
\bar{x}=x^{*}=\Pi_{S}\left(x_{0}\right) .
$$

Thus, the sequence $\left\{x_{i}\right\}$ has a unique cluster point $\Pi_{S}\left(x_{0}\right)$, which shows the global convergence of $\left\{x_{i}\right\}$.

Suppose now that $\bar{x}$ is not a solution of the problem (1). We show first that $k_{i}$ in Algorithm 2 cannot tend to $\infty$. Since $T$ is continuous with compact values, Proposition 3.11 in [26] implies that $\left\{T\left(x_{i}\right): i \in N\right\}$ is a bounded set, and so the sequence $\left\{w_{i}\right\}$ is bounded. Therefore, there exists a subsequence $\left\{w_{i_{j}}\right\}$ converging to $\bar{w}$. Since $T$ is upper semicontinuous with compact values, Proposition 3.7 in [26] implies that $T$ is closed, and so $\bar{w} \in T(\bar{x})$. By the definition of $k_{i}$, we have

$$
\begin{array}{r}
\left\langle u_{i}, r_{\mu}\left(x_{i}, w_{i}\right)\right\rangle<\sigma\left\|r_{\mu}\left(x_{i}, w_{i}\right)\right\|^{2}, \\
\forall u_{i}=\prod_{T\left(x_{i}-\gamma^{k_{i}-1} r_{\mu}\left(x_{i}, w_{i}\right)\right)}\left(w_{i}\right) .
\end{array}
$$

If $k_{i_{j}} \rightarrow \infty$, then $x_{i_{j}}-\gamma^{k_{i_{j}}-1} r_{\mu}\left(x_{i_{j}}, w_{i_{j}}\right) \rightarrow \bar{x}$. The lower continuity of $T$, in turn, implies the existence of $\bar{w}_{i_{j}} \in$ $T\left(x_{i_{j}}-\gamma^{k_{i_{j}}-1} r_{\mu}\left(x_{i_{j}}, w_{i_{j}}\right)\right)$ such that $\bar{w}_{i_{j}}$ converges to $\bar{w}$. Since $u_{i_{j}}=\Pi_{T\left(x_{i_{j}}-\gamma^{k_{i j}-1} r_{\mu}\left(x_{i_{j}}, w_{i_{j}}\right)\right)}\left(w_{i_{j}}\right)$, we have $u_{i_{j}} \in T\left(x_{i_{j}}-\right.$ $\left.\gamma^{k_{i_{j}}-1} r_{\mu}\left(x_{i_{j}}, w_{i_{j}}\right)\right)$ and $\left\|u_{i_{j}}-w_{i_{j}}\right\| \leq\left\|\bar{w}_{i_{j}}-w_{i_{j}}\right\|$. Therefore $\lim _{j \rightarrow \infty} u_{i_{j}}=\bar{w}$ and

$$
\left\langle u_{i_{j}}, r_{\mu}\left(x_{i_{j}}, w_{i_{j}}\right)\right\rangle<\sigma\left\|r_{\mu}\left(x_{i_{j}}, w_{i_{j}}\right)\right\|^{2} .
$$


Letting $j \rightarrow \infty$ in (38), we have

$$
\left\langle\bar{w}, r_{\mu}(\bar{x}, \bar{w})\right\rangle \leq \sigma\left\|r_{\mu}(\bar{x}, \bar{w})\right\|^{2},
$$

with $r_{\mu}(\cdot, \cdot)$ being continuous. It follows from Lemma 5 that

$$
\mu^{-1}\left\|r_{\mu}(\bar{x}, \bar{w})\right\|^{2} \leq \sigma\left\|r_{\mu}(\bar{x}, \bar{w})\right\|^{2} .
$$

Thus, we obtain the contradiction because $\mu<1 / \sigma$. Therefore, $\left\{k_{i}\right\}$ is bounded and so is $\left\{\eta_{i}\right\}$.

By (31) and the boundedness of $\left\{\eta_{i}\right\}$, we obtain that $\lim _{i \rightarrow \infty}\left\|r_{\mu}\left(x_{i}, w_{i}\right)\right\|=0$. Since $r_{\mu}(\cdot, \cdot)$ is continuous and the sequences $\left\{x_{i}\right\}$ and $\left\{w_{i}\right\}$ are bounded, there exists an accumulation point $(\bar{x}, \bar{w})$ of $\left\{\left(x_{i}, w_{i}\right)\right\}$ such that $r_{\mu}(\bar{x}, \bar{w})=$ 0 . This implies that $\bar{x}$ solves the variational inequality (1). Similar to the preceding proof, we obtain that $\left\{x_{i}\right\}$ globally converges to $\bar{x}=\Pi_{S}\left(x_{0}\right)$.

Remark 13. In [11], the mapping $F$ is required to be pseudomonotone. Since the pseudomonotonicity implies condition (3), our assumptions of the mapping $T$ are more general.

Theorem 14. Let $T: K \rightarrow 2^{\mathbb{R}^{n}}$ be continuous with nonempty compact convex values on $K$ satisfying condition (3). Suppose that Algorithm 2 generates an infinite sequence $\left\{x_{i}\right\}$. Then the solution set $S$ of $G V I(T, K)$ is empty if and only if the sequence generated by Algorithm 2 diverges.

Proof. In view of Theorem 12, it is sufficient to prove that if the solution set is empty, then the sequence generated by Algorithm 2 diverges. Since inequality (26) also holds in this case, the sequence $\left\{\left\|x_{i}-x_{0}\right\|\right\}$ is still nondecreasing. We claim that

$$
\lim _{i \rightarrow \infty}\left\|x_{i}-x_{0}\right\|^{2}=\infty .
$$

Otherwise, $\left\{x_{i}\right\}$ is bounded, and hence it follows from (26) that

$$
\lim _{i \rightarrow \infty}\left\|x_{i+1}-x_{i}\right\|^{2}=0 .
$$

A similar discussion as in Theorem 12 would lead to the conclusion that every cluster point of $\left\{x_{i}\right\}$ is a solution of $\operatorname{GVI}(T, K)$, which contradicts the emptiness of the solution set to $\operatorname{GVI}(T, K)$.

\section{Numerical Experiments}

In this section, we present some numerical experiments for the proposed algorithm. The MATLAB codes are run on a PC (with CPU Intel P-T2390) under MATLAB Version 7.0.1.24704(R14) Service Pack 1. We compare the performance of our Algorithm 2 and the algorithms in [5, 11, 12, 22]. In Tables 1, 2, 3, and 4, "It." denotes number of iteration, and "CPU" denotes the CPU time in seconds. The tolerance $\varepsilon$ means that when $\left\|r_{\mu}(x, w)\right\| \leq \varepsilon$, the procedure stops.

Example 15. Let $n=3$,

$$
K:=\left\{x \in \mathbb{R}_{+}^{n}: \sum_{i=1}^{n} x_{i}=1\right\},
$$

\begin{tabular}{|c|c|c|c|c|}
\hline \multirow{2}{*}{$\mathcal{\varepsilon}$} & \multicolumn{2}{|c|}{ Algorithm 2} & \multicolumn{2}{|c|}{$[12$, Algorithm 1] } \\
\hline & It. (no.) & CPU (Sec.) & It. (no.) & CPU (Sec.) \\
\hline $10^{-7}$ & 23 & 0.34375 & 35 & 0.375 \\
\hline $10^{-5}$ & 17 & 0.3125 & 25 & 0.3125 \\
\hline $10^{-3}$ & 10 & 0.265625 & 15 & 0.28125 \\
\hline
\end{tabular}

TABLE 1: Example 15.

\begin{tabular}{lcccc}
\hline$\varepsilon$ & \multicolumn{2}{c}{ Algorithm 2 } & \multicolumn{2}{c}{ [5, Algorithm 1] } \\
& It. (no.) & CPU (Sec.) & It. (no.) & CPU (Sec.) \\
\hline $10^{-7}$ & 23 & 0.34375 & 80 & 1.01563 \\
$10^{-5}$ & 17 & 0.3125 & 57 & 0.828125 \\
$10^{-3}$ & 10 & 0.265625 & 34 & 0.5625 \\
\hline
\end{tabular}

TABLE 2: Example 15.

TABle 3: Example 15.

\begin{tabular}{lcccc}
\hline$\varepsilon$ & \multicolumn{2}{c}{ Algorithm 2 } & \multicolumn{2}{c}{ [22, Algorithm 2.2] } \\
& It. (no.) & CPU (Sec.) & It. (no.) & CPU (Sec.) \\
\hline $10^{-7}$ & 23 & 0.34375 & 27 & 0.390625 \\
$10^{-5}$ & 17 & 0.3125 & 21 & 0.375 \\
$10^{-3}$ & 10 & 0.265625 & 14 & 0.3125 \\
\hline
\end{tabular}

TABLE 4: Example 15.

\begin{tabular}{lcccc}
\hline \multirow{\varepsilon}{*}{} & \multicolumn{2}{c}{ Algorithm 2 } & \multicolumn{2}{c}{ [11, Algorithm 3.1] } \\
& It. (no.) & CPU (Sec.) & It. (no.) & CPU (Sec.) \\
\hline $10^{-7}$ & 23 & 0.34375 & 190 & 1.15625 \\
$10^{-5}$ & 17 & 0.3125 & 134 & 0.875 \\
$10^{-3}$ & 10 & 0.265625 & 79 & 0.5625 \\
\hline
\end{tabular}

and $T: K \rightarrow 2^{\mathbb{R}^{n}}$ be defined by

$$
T(x):=\left\{\left(t, t-x_{1}, t-x_{2}\right): t \in[0,1]\right\} .
$$

Then, the set $K$ and the mapping $T$ satisfy the assumptions of Theorem 12, and $(0,0,1)$ is a solution of the multivalued variational inequality. Example 15 is tested in $[5,12,22]$. We choose $\sigma=0.8, \gamma=0.6$, and $\mu=1$ for our algorithm and Algorithm 1 in [5]; $\sigma=0.4, \gamma=0.9$, and $\mu=1$ for Algorithm 1 in [12]; $\sigma=4, \gamma=0.8$, and $\mu=0.01$ for Algorithm 1 in [22]; $\sigma=0.9, \gamma=0.4$ for Algorithm 3.1 in [11]. We use $x_{0}=(0,1,0)$ as the initial point (Tables $\left.1-4\right)$.

\section{Acknowledgments}

This work is partially supported by Natural Science Foundation Project of CQ CSTC (no. 2010BB9401), Science and Technology Project of Chongqing Municipal Education Committee of China (no. KJ110509), and Foundation of Chongqing University of Posts and Telecommunications for the Scholars with Doctorate (no. A2012-04). 


\section{References}

[1] T. Q. Bao and P. Q. Khanh, "A projection-type algorithm for pseudomonotone nonlips-chitzian multivalued variational inequalities, in generalized convexity,generalized monotonicity and applications," in Proceedings of the 7th International Symposium on Generalized Convexity and Generalized Monotonicity, A. Eberhard, N. Hadjisavvas, and D. T. Lus, Eds., pp. 113-129, Springer, 2005.

[2] Y. Censor, A. Gibali, and S. Reich, "Extensions of Korpelevich's extragradient method for the variational inequality problem in Euclidean space," Optimization, vol. 61, no. 9, pp. 1119-1132, 2012.

[3] F. Facchinei and J.-S. Pang, Finite-Dimensional Variational Inequalities and Complementary Problems, Springer, New York, NY, USA, 2003.

[4] Y. He, "A new double projection algorithm for variational inequalities," Journal of Computational and Applied Mathematics, vol. 185, no. 1, pp. 166-173, 2006.

[5] F. Li and Y. He, "An algorithm for generalized variational inequality with pseudomonotone mapping," Journal of Computational and Applied Mathematics, vol. 228, no. 1, pp. 212-218, 2009.

[6] M. V. Solodov and B. F. Svaiter, "A new projection method for variational inequality problems," SIAM Journal on Control and Optimization, vol. 37, no. 3, pp. 765-776, 1999.

[7] D. Sun, "A class of iterative methods for solving nonlinear projection equations," Journal of Optimization Theory and Applications, vol. 91, no. 1, pp. 123-140, 1996.

[8] Y. J. Wang, N. H. Xiu, and J. Z. Zhang, "Modified extragradient method for variational inequalities and verification of solution existence," Journal of Optimization Theory and Applications, vol. 119, no. 1, pp. 167-183, 2003.

[9] E. Allevi, A. Gnudi, and I. V. Konnov, "The proximal point method for nonmonotone variational inequalities," Mathematical Methods of Operations Research, vol. 63, no. 3, pp. 553-565, 2006.

[10] A. Auslender and M. Teboulle, "Lagrangian duality and related multiplier methods for variational inequality problems," SIAM Journal on Optimization, vol. 10, no. 4, pp. 1097-1115, 2000.

[11] H. Chen, "A new extragradient method for generalized variational inequality in Euclidean space," Fixed Point Theory and Applications, vol. 2013, article 139, 2013.

[12] C. Fang and Y. He, "A double projection algorithm for multivalued variational inequalities and a unified framework of the method," Applied Mathematics and Computation, vol. 217, no. 23, pp. 9543-9551, 2011.

[13] S. C. Fang and E. L. Peterson, "Generalized variational inequalities," Journal of Optimization Theory and Applications, vol. 38, no. 3, pp. 363-383, 1982.

[14] M. Fukushima, "The primal Douglas-Rachford splitting algorithm for a class of monotone mappings with application to the traffic equilibrium problem," Mathematical Programming A, vol. 72, no. 1, pp. 1-15, 1996.

[15] Y. He, "Stable pseudomonotone variational inequality in reflexive Banach spaces," Journal of Mathematical Analysis and Applications, vol. 330, no. 1, pp. 352-363, 2007.

[16] R. Saigal, "Extension of the generalized complementarity problem," Mathematics of Operations Research, vol. 1, no. 3, pp. 260266, 1976.

[17] G. Salmon, J.-J. Strodiot, and V. H. Nguyen, "A bundle method for solving variational inequalities," SIAM Journal on Optimization, vol. 14, no. 3, pp. 869-893, 2003.
[18] M. V. Solodov and B. F. Svaiter, "Forcing strong convergence of proximal point iterations in a Hilbert space," Mathematical Programming A, vol. 87, no. 1, pp. 189-202, 2000.

[19] R. T. Rockafellar, "Monotone operators and the proximal point algorithm," SIAM Journal on Control and Optimization, vol. 14, no. 5, pp. 877-898, 1976.

[20] I. V. Konnov, "On the rate of convergence of combined relaxation methods," Izvestiya Vysshikh Uchebnykh Zavedeniu. Matematika, vol. 37, no. 12, pp. 89-92, 1993.

[21] I. V. Konnov, Combined Relaxation Methods for Variational Inequalities, Springer, Berlin, Germany, 2001.

[22] C. J. Fang and Y. R. He, "An extragradient method for generalized variational inequality," Pacic Journal of Optimization, vol. 9, no. 1, pp. 47-59, 2013.

[23] C. J. Fang, S. L. Chen, and C. D. Yang, "An algorithm for solving multi-valued variational inequality," Journal of Inequalities and Applications, vol. 2013, article 218, 2013.

[24] S. Karamardian, "Complementarity problems over cones with monotone and pseudomonotone maps," Journal of Optimization Theory and Applications, vol. 18, no. 4, pp. 445-454, 1976.

[25] E. H. Zarantonello, "Projections on convex sets in Hilbert space and spectral theory," in Contributions to Nonlinear Functional Analysis, E. H. Zarantonello, Ed., Academic Press, New York, NY, USA, 1971.

[26] J.-P. Aubin and I. Ekeland, Applied Nonlinear Analysis, John Wiley \& Sons, New Yor, NY, USA, 1984. 


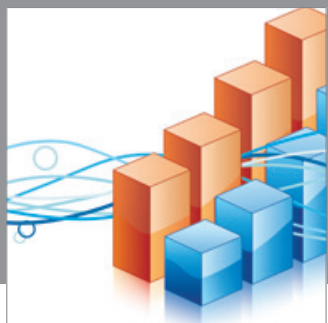

Advances in

Operations Research

mansans

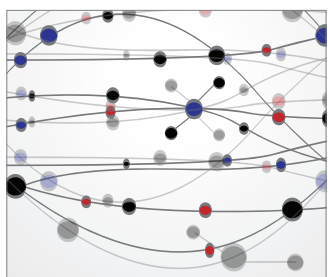

The Scientific World Journal
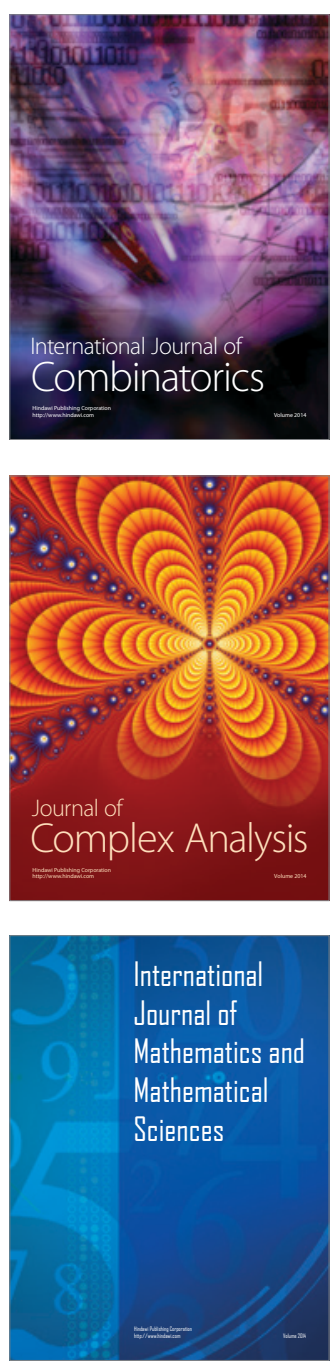
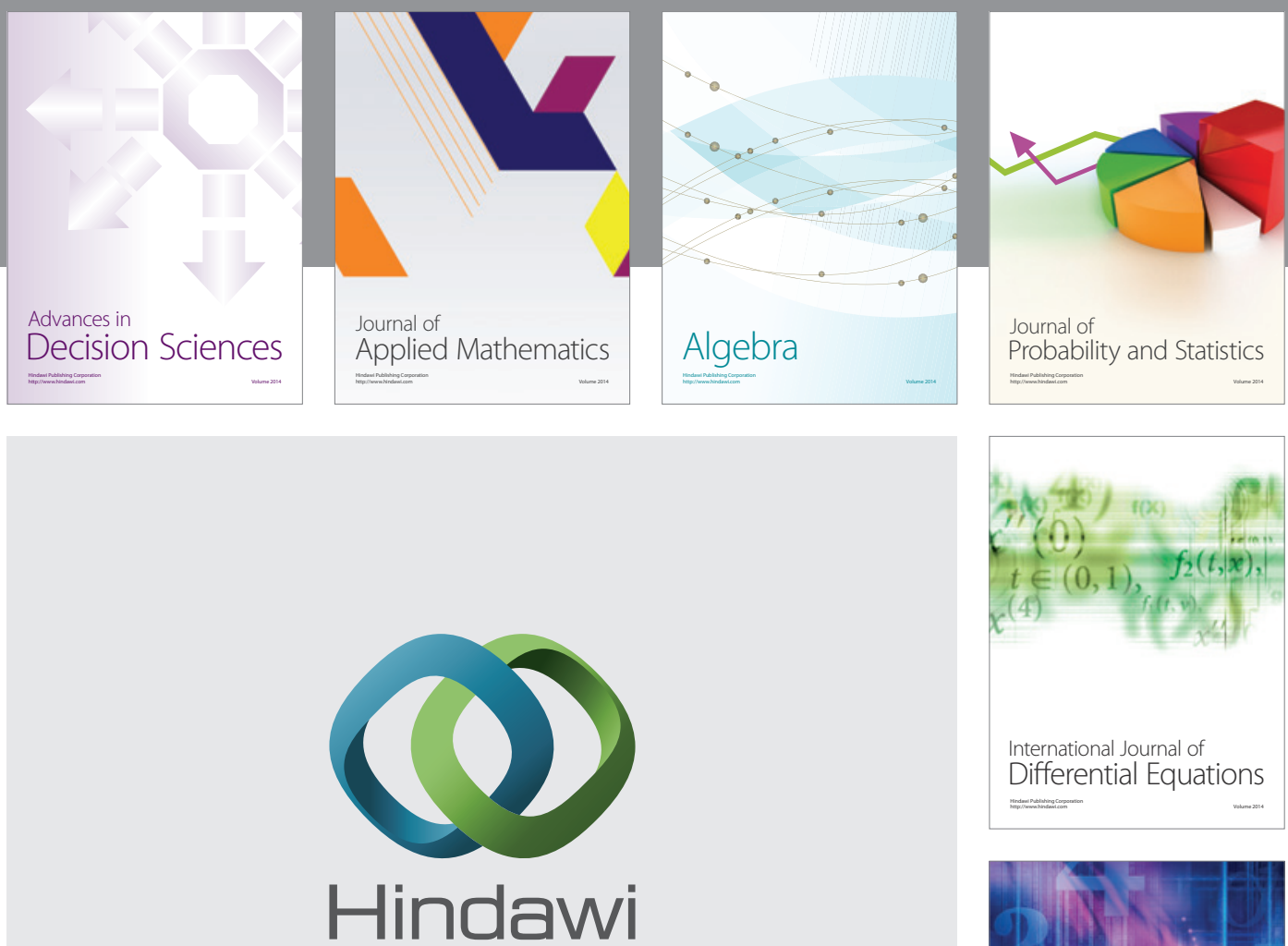

Submit your manuscripts at http://www.hindawi.com
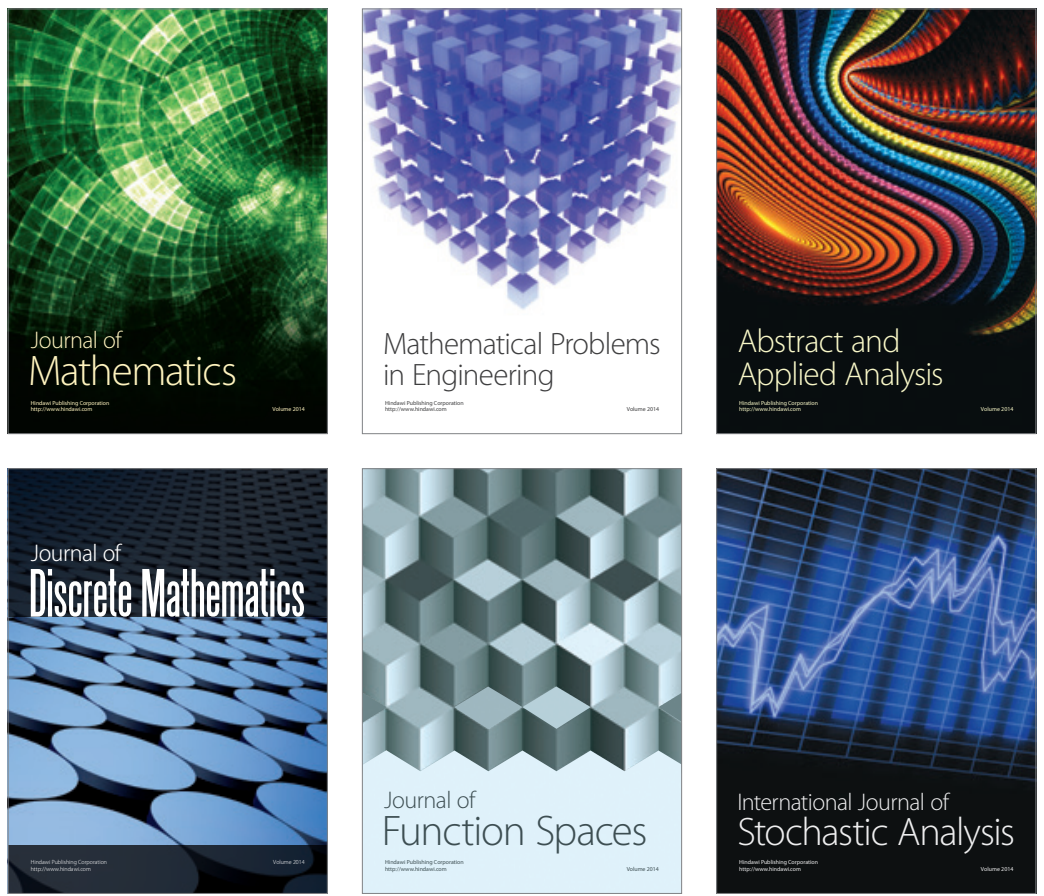

Journal of

Function Spaces

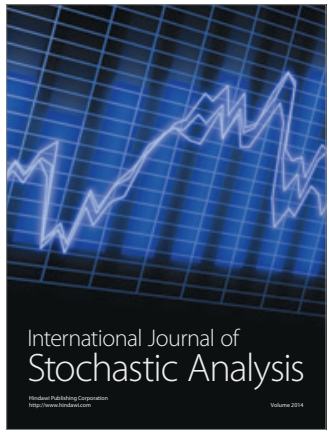

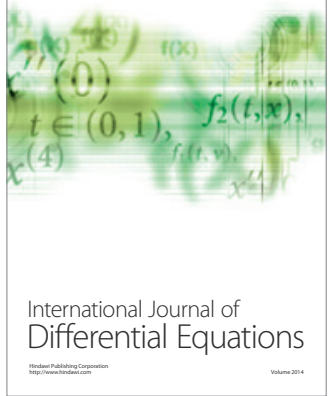
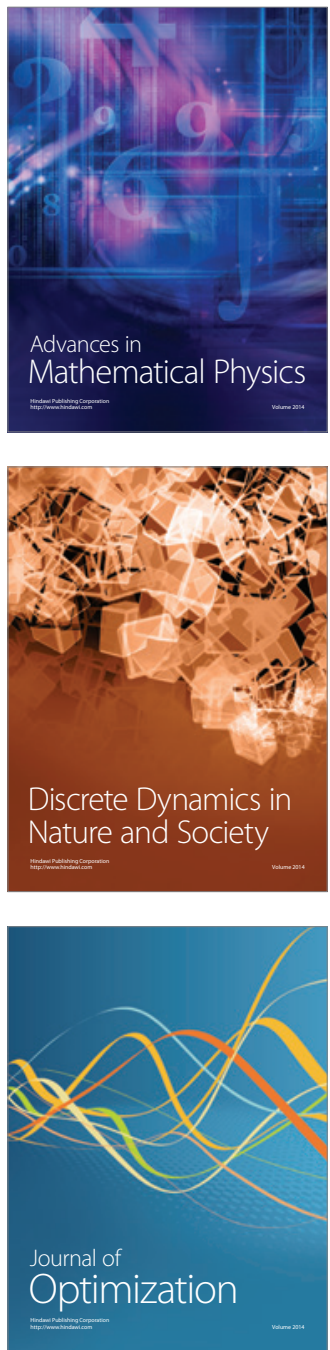